\title{
PAISAJES Y PRODUCCIÓN EN LA HISTORIA. ENFOQUES Y MÉTODOS
}

\author{
Landscapes and Production in History. \\ Approaches and Methods
}

\author{
Cristina NOVOA JÁUREGUI \\ Universidad de Cantabria \\ E-mail: cristina.novoa@unican.es
}

Fecha de recepción: $19-02-2010$

Fecha de aceptación: 22-02-2010

RESUMEN: Esta ponencia se plantea como reflexión general, a partir de dos casos, del análisis del paisaje en dos momentos históricos dispares, respondiendo a estímulos igualmente dispares, pero que comparten su preocupación en las transformaciones del medio como consecuencia de la producción del hombre, en el seno de una Arqueología que se consolida, en nuestro país, como ciencia autónoma, ciencia social.

Palabras Clave:Arqueología, paisaje, producción, investigación, gestión, perspectivas, época clásica, época contemporánea.

ABSTRACT: This paper is presented as a general consideration, from two cases, about the analysis of the landscape in two different historical moments, responding to stimuli equally mixed, but sharing its concern at the environmental changes resulting from the production of man, in the Archeology within a consolidating, in our country, as an autonomous science, social science.

Keywords: Archaeology, Landscape, Academic Archaeology, Commercial Archaeology, Views, Classic Period, Contemporary Period 


\section{INTRODUCCIÓN.}

Cuando se planteó la posibilidad de presentar una ponencia en este primer Encuentro Interdisciplinar de Jóvenes Investigadores, consideré que ésta podía ser la oportunidad para recapacitar, de forma general, sobre el rumbo que los trabajos sobre "eso" que denominamos "paisaje" están siguiendo en diferentes esferas -académica, de gestión-, preocupándose por diferentes momentos históricos o facies y contrastando dos casos vinculados a la actividad productiva humana. El corpus defendido como metodología idónea en el análisis de las transformaciones antrópicas del medio, a lo la largo del tiempo, por la interacción de los hombres con la naturaleza (o paisaje), se relativiza en la particularidad de los casos. Esto es, deudora de una formación clásica mediterránea de análisis del territorio de ciudades y su hinterland-prospección intensiva, morfohistoria, recurso o al menos propuesta de técnicas de análisis de las condiciones ambientales- el enfrentamiento a otros marcos de actuación ha supuesto el punto de partida de la reconsideración de determinados presupuestos. Por tanto, la presente ponencia es única y exclusivamente, una breve reflexión sobre nuestro propio trabajo de investigación previo y nuevos campos sugerentes que nos interesan, como consecuencia de nuestra corta trayectoria profesional y concernientes a cómo se estudia "Arqueología del Paisaje" y "Arqueología de la Producción”, más bien los contextos en que ambas disciplinas están imbricadas.

La preocupación por el análisis desde la Arqueología de "las manufacturas con el fin de extraer elementos para la reconstrucción de las relaciones de los hombres entre sí y de los hombres entre las cosas, en las diversas situaciones históricas" (MANNONI, GIANICHEDA 2004) y cómo dichas relaciones dejan su huella material en el paisaje, se debe, en parte, a la valoración de su importancia en el cambio cultural. Hemos introducido varios conceptos claves; manufacturas, relaciones sociales, historia, paisaje y cambio. Queremos llamar la atención al hecho de que, en una única oración, se han podido vincular a dichos significantes, cargados de significado, de una manera coherente, sin altisonancias; síntoma inequívoco de la asunción, por nuestra parte, de que éstos se interrelacionan de acuerdo a los parámetros de discernimiento en los que nos movemos. La tecnología o el conjunto de saberes (logos), que permiten con arte o técnica (teknos) producir dichas manufacturas, no es un medio para un fin meramente adaptativo. Igualmente el "lugar" (site place) en que se desarrollan dichas actividades no es el escenario físico aprehendido objetivamente, que impone límites a su explotación y por ende, a la evolución social (DAVID, THOMAS 2008). Estas interpretaciones se derivan de que, simple y complicadamente, la Arqueología devuelve la dimensión compleja de "lo social" a los restos materiales, diversos en tiempo y lugar, independientemente de la deuda que el arqueólogo(s) tenga, de conceptuaciones históricas, antropológicas etc. De hecho, este congreso se presenta 
como una ocasión idónea para debatir la línea que, desde la base, entiéndase, jóvenes investigadores, están tomando las diversas disciplinas de las ciencias sociales. No nos sorprendería que tuviéramos más puntos en común de los que consideramos a priori, como consecuencia de la formación compartimentada que hemos recibido y reproducimos, a veces, por inercia.

La conciencia de la necesidad de comprender las relaciones sociales desde el resto arqueológico, e incluimos dentro de resto arqueológico el paisaje como un todo, más allá de esquemas descriptivos y mecanicistas, no significa que siempre sea posible. El estadio de la investigación en el que nos encontremos, los fines que persigamos o que nos impongan, entre otros, han de ser tenidos en cuenta, como ejemplificaremos. Aspiración de máximos que ha de construirse desde mínimos logrados y muchas veces esta operación, se nos antoja complicada.

No queremos dejar escapar tampoco la ocasión de hacer un breve apunte sobre el papel dinámico, reconocido más en el extranjero que en nuestro país, que ha tenido la profesionalización para la reinterpretación de la Arqueología y en el caso concreto que nos compete, la disciplina de paisaje dentro de la misma. Uno de sus principales aportes, apuntado por otros especialistas, es el contacto con realidades pluriestratificadas que analizar y el paisaje, se define como tal (DAVID, THOMAS 2008). La arqueología profesional no entiende de la mayor o menor importancia de la domus romana con respecto del cuartel del s. XIX en un yacimiento, ni de la prospección "diacrónica temática", ni de que las evidencias estén enterradas, en superficie o construidas. Entiende de "productos culturales que tienen que socializarse", o al menos hacia tal intenta dirigirse (BARREIRO 2005). Cabe preguntarse, pues, a modo de conclusión de estas líneas introductorias, si no nos deberíamos replantear cuál es nuestra función como arqueólogos.

2. Nuestra EXPERIENCIA. El TERritorio DE Tricio y los ALFARES DE TERRA SIGILLATA HISPÁNICA.

Nuestro estudio del territorio de Tricio puede adscribirse al marco de análisis "diacrónico-temático" al que hemos aludido líneas arriba. El ser concebido como un proyecto de investigación encuadrado en una beca de investigación predoctoral, propició una situación favorable para la reflexión metodológica en el análisis de una arqueología de asentamientos productivos que, tal vez, en otro contexto, no habría sido posible (NOVOA en prensa, 2009, 2006; ARIÑO, NOVOA, 2007).

La vuelta a los niveles regionales de análisis del territorio, en que se prospectan intensivamente determinados transectos, por parte de equipos de investigación, algunos de los cuales incluyen ya en sus fines la gestión y 


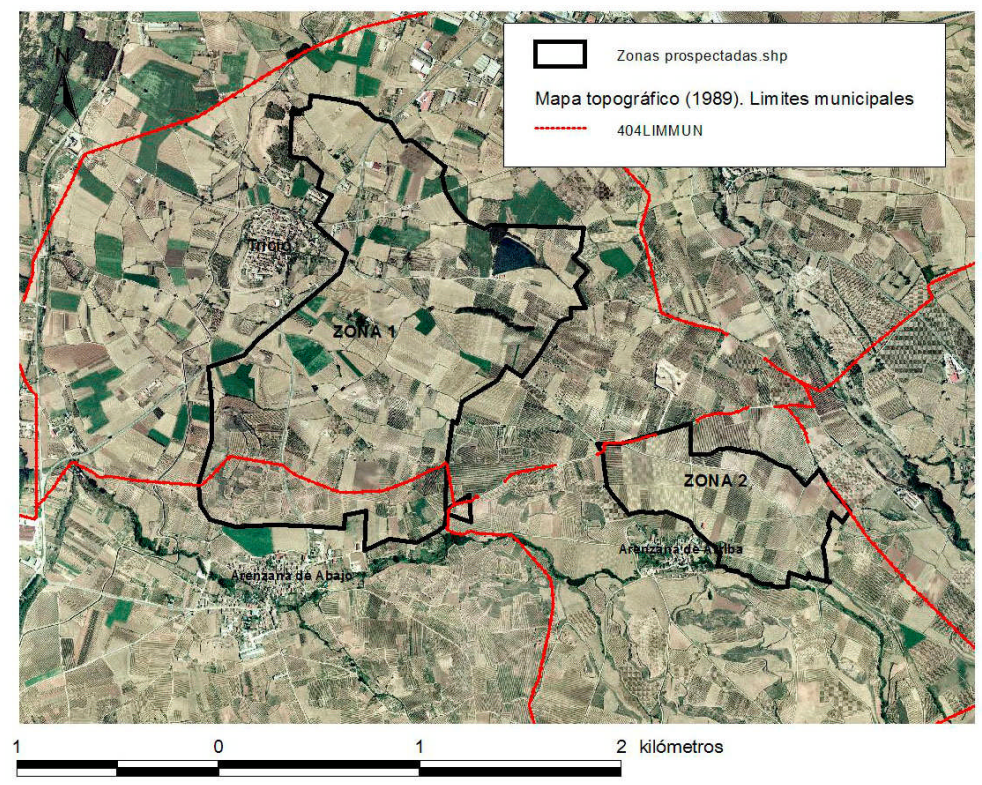

Fig. 1 Zonas de prospección, de acuerdo al sistema de cobertura total y la parcela como unidad de campo.

preservación del patrimonio, están marcando una tendencia divergente (GARCÍA SANJUÁN, VARGAS DURÁN, WHEATLEY 2004). Nosotros optamos por una prospección intensiva de cobertura total de dos áreas dentro de una microrregión que, si bien supuso una inversión temporal elevada, nos sirvió de campo de experimentación de un método en un caso concreto, más allá de los resultados. De acuerdo a la finalidad de esta ponencia, no nos interesan tanto estos últimos per se, como las conclusiones que extrajimos de la aplicación de dicha metodología de acuerdo a nuestra "finalidad de paisaje".

La prospección intensiva fue el eje en torno al que se articuló otra serie de técnicas de diversas disciplinas, cuya aplicación perseguía conocer las transformaciones que el entorno de la ciudad romana de Tricio había experimentado con el establecimiento de unos alfares. Interpretados éstos como "una manifestación antrópica-económica de cambio en un periodo histórico concreto", perseguimos, en primer lugar y ante todo, determinar dónde se localizaban y cuál era su patrón de distribución espacial (prospección arqueológica, prospección aérea y prospección geofísica), explicándolo de acuerdo a unas hipótesis preliminares acerca de las posibles fuentes de extracción de arcilla (análisis arqueométricos) y macroestructuras antrópicas del territorio (fuentes medievales) (NOVOA 2009). El peso recayó en el primero de los 
puntos, esto es, en la constatación y descripción de un determinado modelo de distribución de los centros de producción en la periferia suburbana de Tritium; la discusión en torno a los problemas de campo, de gestión y análisis de los datos, que podría traer consigo - de hecho las trajo- el diseño de una prospección con técnicas intensivas en un medio de tales características culturales, se convirtió en el epicentro del trabajo. Traducida la periferia suburbana en un continuo de material en superficie, nuestra investigación se dirigió en torno a las variables que habíamos de tener en cuenta para clasificar y correlacionar contextos superficiales con determinas formas de "vivir" en el pasado. Se reveló una complejidad distributiva de los alfares desconocida hasta el momento que hubo que explicar después y que, a día de hoy, seguimos teniendo que explicar si queremos, como hemos expuesto en la introducción, inferir la manera en que se organizan los hombres, en nuestro caso, en relación con la producción de terra sigillata. Introducimos la problemática de la localización de las fuentes de extracción de arcilla y la cuestión de la gestión hídrica y articulación de la red viaria. Nuestro objetivo era sentar unas bases, de cara al futuro, que nos sirvieran para construir hipótesis cada vez más argumentadas acerca de la influencia de condiciones materiales de recursos y ordenación del territorio en la distribución microregional de los alfares y en última instancia, si esa organización podría traducirse en términos de organización social. Dejamos, a su vez, en el aire, la pregunta de la elección de ese enclave para ubicar unos alfares de terra sigillata en el contexto expansivo del Imperio romano, abogando por la necesidad de un enfoque comparativo de lo acaecido en otras provincias de forma escalonada.

En conclusión, la Arqueología de Paisaje y Arqueología de Producción se tradujo, por nuestra parte, en la exigencia primaria de definir unos métodos de análisis de un caso el cual, hasta el momento, no había sido estudiado desde este enfoque. Así, tuvimos que preguntarnos acerca de la viabilidad, potencialidad y déficits de un diseño y técnicas de prospección; el ámbito de investigación en que nació el proyecto fue propicio para ello. Planteamos además vías futuras de exploración, tras una inicial compilación por nuestra parte de determinada información ${ }^{1}$, con el fin no tanto de hacer historia, sino de comprender el comportamiento de las sociedades humanas y cómo éste se traduce en donde viven, desde los mecanismos metodológicos e interpretativos, propios y compartidos, de la Arqueología como Ciencia Social.

\section{OTRAS EXPERIENCIAS.}

El paisaje industrial es, sin duda, una de los más recientes e interesantes campos que están desarrollándose y "se opone" diametralmente, en principio, al espíritu conforme al que nació, por ejemplo, el proyecto de Tricio o experiencias similares en otras áreas. 
El contacto con el mismo nace de un trabajo pequeño y secundario en el ámbito profesional, que se calificaría incluso de urgencia ${ }^{2}$. Este supuso un estímulo personal para el inicio de una reflexión acerca de cómo se estudiaba el paisaje del que esas estructuras, que iban a ser derruidas, formaban parte. La expansión urbana, el desarrollo y ampliación del concepto de bien con interés patrimonial y la progresiva toma de conciencia de la agresión, en nuestro país, a elementos de nuestro pasado reciente, pero pasado sin duda alguna, ha repercutido en el surgimiento de medidas de protección y en paralelo, la preocupación por los restos que, en ese espacio, testimonian el modo de producción industrial (VICENTI 2007). Qué es antes, el estímulo de la práctica y su "acción transformadora" o el de la teoría y su "capacidad crítica" es otro interrogante que no nos compete discutir en este momento (BARREIRO 2005). La puesta en marcha, desde el 2000, de un Plan Nacional del Patrimonio Industrial, supone el reconocimiento a nivel nacional de dichos bienes, reconocimiento impulsado previamente desde el ámbito autonómico, en concreto desde aquellas comunidades en las que el proceso de industrialización fue más profundo (IBÁÑEZ M. et alii 1988, CARCAMO 1988; VILLAR 1994)

(http://www.mcu.es/patrimonio/MC/IPHE/PlanesNac/PlanIndustrial/PatrimonioIndustrial.html).

El análisis de la facies contemporánea desde la Arqueología, es todavía embrionario y sin una metodología definida. La propia interpretación generalizada de paisaje es deudora de presupuestos bien urbanistas actuales, bien históricos, más que arqueológicos, ya que, en la base, los restos materiales o huellas del mismo, se conciben desde una perspectiva monumental arquitectónica - artística; situación que recuerda a las experimentadas para otros periodos décadas atrás. Es un ejemplo, en otras palabras, del proceso de autodefinición de la arqueología como ciencia autónoma. Si bien se encuentra, en líneas generales, en un proceso inicial de identificación y clasificación de sus "bienes" -con vistas así mismo a su gestión y conservación de acuerdo al contexto en que nos encontramos actualmente-, las propuestas giran en torno a caracterizar las "fuentes materiales" 3 e integrarlas en el medio, construir su paisaje, atendiendo a los lugares de aprovisionamiento de materias primas, centros para su elaboración etc. (CASADO 2009a, 2009b; VICENTI 2007). Esto es, Arqueología del Paisaje y Arqueología de la Producción, para cuyo análisis debe desarrollarse una metodología arqueológica, cuyas técnicas están en proceso de adaptación al caso de estudio. Por ejemplo, resultaría inviable el diseño de una prospección tal como la que planteamos en el caso de Tricio, pero tal vez sería factible una prospección del espacio construido tal y como se plantea desde la Arqueología de la Arquitectura, la cual está ofreciendo resultados óptimos para el análisis de paisajes de otros momentos, medieval - moderno, como es el caso de la producción tradicional de sal y el 
yacimiento de Salinas de Añana (PLATA 2009) o toda la reflexión en torno al ciclo productivo de la piedra de las ermitas altomedievales (SÁNCHEZ ZUFIAURRE 2007; CABALLERO, UTRERO 2005). Arqueología de la Arquitectura implica la definición cronotipológica de los edificios, atendiendo no a variables (conjuntos de) estilísticas y/o formales sino tecnológicas, como se presupone el análisis tecnológico, por ejemplo, para el estudio de extracción de recursos y su fosilización en las áreas en que se ha dado. Poder vincularlos, así, en una estructuración territorial para la que tenemos acceso a documentación escrita, cartográfica, fotográfica etc. Con el objetivo de comprender en el espacio las relaciones sociales de un determinado periodo. $\mathrm{Si}$ por algo se intenta caracterizar a la Arqueología en su proceso de autoreafirmación en las últimas décadas, es por su intento de ser una ciencia crítica e independiente -en qué medida de todas maneras puede serlo- fortaleciendo su código de actuación.

Resumiendo, el paisaje industrial se trata de una disciplina naciente, en paralelo al reconocimiento legal y en el que se implican agentes administrativos públicos, académicos, profesionales y asociaciones de diversa índole que obliga a la colaboración mutua. Todavía se encuentra en una fase de definición del objeto de estudio y métodos con que abordarlo, vinculado a visiones monumentales-patrimoniales.

\section{CONCLUSIÓN.}

No hemos elegido al azar estas dos "experiencias de paisaje", sino por contrapunto en varios aspectos, repetimos, desde nuestra corta experiencia investigadora y profesional:

En primer lugar, el estímulo que nos ha llevado a preocuparnos por ello y la retroalimentación necesaria de la práctica académica y la práctica profesional.

En segundo lugar, los diversos planteamientos y relacionados con ellos, los trabajos que se plantean. En época clásica, desde hace unas décadas, hay, con más acuerdo que peros, definidos los mínimos respecto a qué es paisaje y cómo se estudia, de manera que muchos trabajos, como el de Tricio, reflexionan sobre determinadas técnicas aplicadas a determinados casos, dependiendo del estado de la investigación y con la persecución de máximos a largo plazo $^{4}$; en época contemporánea, en cambio, estamos asistiendo a una reflexión y proceso de consolidación, tanto del qué como del cómo, ya que, hasta ahora, muchas publicaciones son catálogos de maquinas, fábricas, etc. Paso inicial indispensable en el que nosotros, consideramos, pueden introducirse métodos y será necesario, de hecho, en relación con los espacios construidos. 
En tercer y último lugar, porque ejemplifican el vínculo estrecho entre la Arqueología de la Producción y la Arqueología del Paisaje, etiquetas que más bien definen campos, necesarios, cuando la Arqueología, a secas, está ampliando su radio de acción y erigiéndose como una ciencia autónoma aplicada. Por tanto, y a modo de conclusión final, no podemos olvidar, como en mi propio trabajo de investigación señalé, que el paisaje es una parcela de la misma, y como tal no puede aspirar a comprender la totalidad de la experiencia humana (NOVOA 2009).

\section{BiBLIOGRAFÍA}

ARIÑO E., NOVOA C. 2007, "Fotografía aérea en la Rioja Alta. Tritium Magallum (Tricio) y Libia (Herramelluri)". Revista de Arqueología del s. XXI 315: 54-63.

BARREIRO D. 2006, "Conocimiento y acción en la Arqueología Aplicada". Complutum, Vol. 17: 205-219.

CABALLERO L.; UTRERO M. A. 2005, "Una aproximación a las técnicas constructivas en la Alta Edad Media". Arqueología de la Arquitectura 4: 169-192.

CÁRCAMO J. 1988, Biףkaiko industria ondarea $:$ [erakusketa] $=$ Elpatrimonio industrial de Biøkaia :[exposición]. Bilbao.

CASADO GALVÁN 2009a, "Las fuentes materiales de la Arqueología industrial". Contribuciones a las Ciencias Sociales, diciembre 2009. www.eumed.net/rev/cccss/06/icg13.htm.

CASADO GALVÁN 2009b, "Breve historia de la protección del patrimonio industrial". Contribuciones a las Ciencias Sociales, noviembre 2009. www.eumed.net/rev/cccss/06/icg4.htm

DAVID B., THOMAS J. (eds.) 2008, Handbook of Ldscape Archaeology.California

GARCÍA SANJUÁN L., VARGAS DURÁN M. A. y WHEATLEY D. W. 2004, "Prospecciones de superficie en la zona de afección del embalse de Los Melonares (Almadén de la Plata, El Pedroso y Castilblanco de los Arroyos, Sevilla)." Anuario Arqueológico de Andalucial 2001. Tomo III. Actividades de Urgencia, 962-972. Sevilla. Junta de Andalucía (2004).

IBÁÑEZ M. et alii 1988, Arqueología industrial en Vižcaya. Bilbao.

NOVOA C. en prensa, "Definición de contextos materiales superficiales en áreas alfareras: prospección intensiva en el territorio de Tritium Magallum (La Rioja, España). Actas del $26^{\text {th }}$ congress of the REI CRETARIAE ROMANAE FAUTORES. 
NOVOA C. 2009, Arqueología del Paisaje y producción cerámica: los alfares romanos del valle del Najerilla (La Rioja) y su distribución espacial. Tesis Doctoral inédita. Universidad de Salamanca.

NOVOA C. 2006, "Alfares romanos y territorio. Bases para el estudio del paisaje antiguo del Valle del Najerilla (La Rioja, España)". Agri Centuriati 3: 111-132.

PLATA MONTERO A. 2009, Arqueología de las Salinas. El método de estudio de un paisaje cultural construido. KOBIE (Serie paleoantropología). Bilbao $\mathrm{N}^{\circ}$ XXVIII: 255 -266.

SÁNCHEZ ZUFIAURRE L. 2007, Técnicas constructivas medievales. Nuevos documentos arqueológicos para el estudio de la Alta Edad Media en Alava. EKOB 3.

VICENTI A. 2007, "Perspectivas sobre la Arqueología Industrial”. Arqueoweb 9 (1): 1-49.

VILLAR J. E. 1994, Las catedrales de la industria: patrimonio industrial en la margen izquierda y zona minera de la ría del Nervión. Bilbao.

\section{NoTAS}

${ }^{1}$ No estamos refiriendo, por ejemplo, al vaciado de la documentación medieval, a partir del que extrajimos un listado de infraestructuras y elementos del paisaje que ubicamos espacialmente.

${ }^{2}$ La Arqueología profesional no puede entenderse, a día de hoy, de urgencia -término así mismo, con intencionalidad no muy inocente- sino, más bien, como de gestión, ya que incluso intervenciones residuales pueden ser aprovechadas como motor de investigación.

${ }^{3}$ Fábrica, máquina, vivienda obrera, vías de comunicación y transporte.

${ }^{4}$ Cada vez somos más conscientes de que estudiar paisaje, además, implica empezar por parcelas acotadas. 